\title{
Análisis de isótopos estables en restos óseos humanos y de fauna en los sitios arqueológicos del Holoceno temprano y medio Tequendama y Aguazuque (sabana de Bogotá, Colombia) roseach phaser
}

$$
\begin{aligned}
& \text { Analysis of stable isotopes in human and wildlife skeletal remains } \\
& \text { in the archeological sites of the early and middle Holocene } \\
& \text { Tequendama and Aguazuque (savanna of Bogotá, Colombia) }
\end{aligned}
$$

\author{
Angélica Viviana Triana Vega ${ }^{1}$ \\ Universidad de los Andes \\ Universidad Externado de Colombia \\ (iD https://orcid.org/0000-0002-6943-7839 \\ Isabel Casar ${ }^{3}$ \\ Universidad Nacional Autónoma de México \\ (iD https://orcid.org/0000-0001-9352-9197
}

\author{
Pedro Morales ${ }^{2}$ \\ Universidad Nacional Autónoma de México \\ (iD https://orcid.org/0000-0001-5767-9964
}

\author{
Jennifer Salinas ${ }^{4}$ \\ Universidad de Berkeley \\ (D) https://orcid.org/0000-0001-8530-6452
}

Resumen: Algunos estudios arqueológicos sobre los procesos de cambio social de las sociedades que existieron durante el Holoceno temprano y medio en Colombia han tenido lugar en la sabana de Bogotá (Colombia). La investigación de Gonzalo Correal y Thomas van der Hammen en los sitios arqueológicos de Tequendama y Aguazuque son ejemplos relevantes. Estos autores recopilaron información importante de artefactos líticos, restos de plantas, alfarería y esqueletos humanos y de animales, que proporcionan datos sobre las formas de vida de los humanos que habitaron estos sitios. En 2014, un nuevo estudio se centró en la reexcavación de los sitios de Tequendama y Aguazuque a través de nueve columnas de 50 x $50 \mathrm{~cm}$. Esta excavación se llevó a cabo para obtener información arqueobotánica e identificar el acceso a los recursos que poseían los pobladores de estos dos sitios. Este documento presentará los resultados del análisis de isótopos estables obtenidos con base en restos óseos humanos y de fauna, para aproximarnos a la dieta de algunos de los habitantes que ocuparon los sitios arqueológicos de Tequendama y Aguazuque, los cuales presentan una ocupación entre el Holoceno temprano y medio, y la transición de la caza y recolección a los primeros procesos de horticultura y agricultura en dichos sitios.

Palabras clave: acceso a recursos, cazadores-recolectores, dieta, Holoceno, isótopos estables.

Recibido: 28/02/2019 | Aceptado: 17/02/2020 | Disponible en línea: 4/03/2020

Como citar este artículo: Triana-Vega, A. V., Casar, I., Morales, P. y Salinas, J. (2020). Análisis de isótopos estables en restos óseos humanos y de fauna en los sitios arqueológicos del Holoceno temprano y medio Tequendama y Aguazuque (sabana de Bogotá, Colombia): Jangwa Pana, 19(1), 10 - 22. Doi: https://doi.org/10.21676/16574923.3432

${ }^{1}$ Correo electrónico: angelica.triana@uexternado.edu.co; av.triana177@uniandes.edu.co

${ }^{2}$ Correo electrónico: pedro_morales1@icloud.com

${ }^{3}$ Correo electrónico: casar_isabel@yahoo.com

${ }^{4}$ Correo electrónico: salinas@berkeley.edu 
Abstract: Some archaeological studies on the processes of social change of the societies that existed during the early and middle Holocene in Colombia have taken place in the Sabana de Bogotá (Colombia). The research carried out by G. Correal and T. van der Hammen in the archaeological sites of Tequendama and Aguazuque are relevant examples. These authors collected important information on lithic artifacts, plant remains, pottery and human and animal skeletons that provide information on the lifestyles of the humans who inhabited these sites. In 2014, a new study focused on the re-excavation of the Tequendama and Aguazuque sites through nine $50 \times 50 \mathrm{~cm}$ columns. This document will present the results obtained from the analysis of stable isotopes obtained from human and wildlife bone remains to approximate the diet of some of the inhabitants who occupied the archaeological sites of Tequendama and Aguazuque, which present an occupation between the Early and middle Holocene and the transition from hunting and gathering to the first processes of horticulture and agriculture in these sites.

Key words: Hunter and Gatherers; Holocene; Stable isotopes; Diet; Access to resources

\section{Introducción}

Los estudios arqueológicos en la sabana de Bogotá han logrado reconstruir parte de la información sobre las formas de vida y subsistencia de sociedades preagrícolas en Colombia. Dos hitos han sido las excavaciones en Tequendama y Aguazuque. Gracias a las excavaciones realizadas en el primer lugar en los años setenta, Gonzalo Correal y Thomas van der Hammen reportaron una ocupación desde finales del Pleistoceno hasta el Holoceno tardío. En el sitio hay presencia de artefactos líticos, restos óseos humanos y fauna, además de restos vegetales fechados aproximadamente entre 10.700 y 10.000 AP (Correal y Van der Hammen, 1977). Aguazuque, por su parte, fue excavado hacia finales de los años ochenta por Gonzalo Correal. En ese lugar se obtuvo una ocupación entre el 8000 y el 2500 AP aproximadamente, y además de encontrar una gran cantidad de entierros, artefactos líticos y restos óseos de fauna, se recuperaron también restos vegetales carbonizados (Correal, 1990).

Los restos de plantas encontrados en ambos sitios y los trabajos basados en el análisis de isótopos estables realizados en los restos humanos (Van der Hammen, Urrego y Klinken, 1990; Cárdenas, 2002; Delgado, 2018) proporcionan información importante acerca de la dieta y los recursos vegetales utilizados por estas poblaciones (Cárdenas, 2002; Delgado, 2017; Van der Hammen, Urrego y Klinken, 1990). Estos trabajos dan una idea bastante detallada de los cambios en la alimentación entre el Holoceno temprano y medio, y constituyen una enorme contribución al conocimiento de este tema en una escala temporal amplia. Sin embargo, aún falta establecer relaciones entre la información sobre subsistencia $\mathrm{y}$ aspectos sociales de las comunidades que ocuparon Tequendama y Aguazuque, especialmente en cuanto al uso y acceso a los recursos en estos periodos. Con base en lo anterior, en este trabajo se reportará la información de datos isotópicos obtenidos en restos óseos humanos y de fauna en la reexcavación de los sitios de Tequendama y Aguazuque, llevada a cabo en 2014 (Triana y Salinas, 2014).

Los sitios Tequendama y Aguazuque muestran la existencia de contextos muy diferentes, aunque en ambos se trata de sociedades preagrícolas. Tequendama se interpreta como un lugar ocupado por sociedades tempranas de cazadores-recolectores, móviles, con un alto consumo de proteína de origen animal. Por su parte, en Aguazuque existen evidencias de una mayor dependencia de plantas y un mayor sedentarismo, probablemente acompañado de una complejidad social más alta (Correal, 1990; Correal y Van der Hammen, 1977). El contraste 
que puede establecerse en cuanto al acceso a recursos en ambos lugares puede dar una idea de los cambios ocurridos en el tránsito entre la caza y la recolección, propias de los habitantes de Tequendama, y los inicios de la horticultura, identificada para Aguazuque. Aunque el tamaño de la muestra es reducido, esta se considera información relevante, puesto que sirve para complementar los datos hallados en otros trabajos (Cárdenas 2000; Van der Hammen, Urrego y Klinken, 1990). Ambos sitios son de particular importancia porque ofrecen evidencias de macrorrestos de plantas, restos humanos de ambos sexos y una serie de fechas de ocupación de los sitios.

\section{Materiales y métodos}

\section{Área de estudio}

En la cordillera oriental de Colombia se encuentra una extensa planicie de altura conocida como la sabana de Bogotá, ubicada en el centro geográfico de Colombia, al sur del altiplano cundiboyacense y a una altura promedio de $2.600 \mathrm{~m} \mathrm{~s}$. n. m., en el departamento de Cundinamarca, municipio de Soacha. Dentro de la sabana de Bogotá se ubican los sitios arqueológicos de Aguazuque y Tequendama, rodeados por los cerros Encantado, Gordo y Mondoñedo (Correal, 1990).

El sitio arqueológico Tequendama se ubica en un abrigo rocoso en las coordenadas $4^{\circ} 31^{\prime} 59,881^{\prime \prime} \mathrm{N}$ y $74^{\circ} 16^{\prime} 30,895^{\prime \prime} \mathrm{W}$, a $446 \mathrm{~m}$ de la entrada principal de la Hacienda Tequendama y orientado en dirección $12^{\circ}$ desde el peaje de Chusacá, en Soacha. Aguazuque es un sitio arqueológico a cielo abierto, ubicado en las coordenadas 4³6'32,200" N y 74 $16^{\prime} 50,375^{\prime \prime}$ W. Se sitúa en el predio privado que desde el siglo XVII hasta hace algunos años era llamado Hacienda Aguazuque. Actualmente este lugar lleva como nombre Hacienda Fute (figura 1).

Para las condiciones ambientales de la sabana de Bogotá se han reportado cambios a lo largo de la transición Pleistoceno/Holoceno y Holoceno en general. En los periodos más tempranos (11.800-10.900 cal BP) se menciona que el clima era frío, que el bosque desaparece y que la vegetación es de subpáramo con biomas de arbustos, áreas abiertas y pastizales (Delgado, 2018; Marchant et al., 2002; Van der Hammen, 1992). Hacia el Holoceno medio, entre cal 8300 cal BP y ca. 6800/5800 cal BP, el clima es seco y frío. Los bosques de Quercus invaden el altiplano y se reducen las zonas secas y abiertas. Hacia el 6800/5800 cal BP, el nivel de los lagos desciende, el clima es más favorable, el nivel total del bosque aumenta y se caracteriza por tener Robles, Podocarpus, Urticaceas y Cecropias. El ambiente tiende a volverse seco para este periodo, y se evidencia una disminución en las capas freáticas (Marchant et al., 2002; Mora y Pratt, 2002). Van der Hammen (1992) reportó que para este periodo hubo una sequía en Tequendama (Delgado, 2012, 2017, 2018). Para periodos tardíos, hacia 2500 cal BP, se registra que el clima era más húmedo. Así mismo, la temperatura disminuye hasta convertirse en la temperatura actual $\left(12{ }^{\circ} \mathrm{C}-18{ }^{\circ} \mathrm{C}\right.$, aproximadamente $)$, y la vegetación está constituida por plantas herbáceas con zonas pantanosas y áreas inundables. 
Figura 1. Mapa de ubicación de los sitios arqueológicos

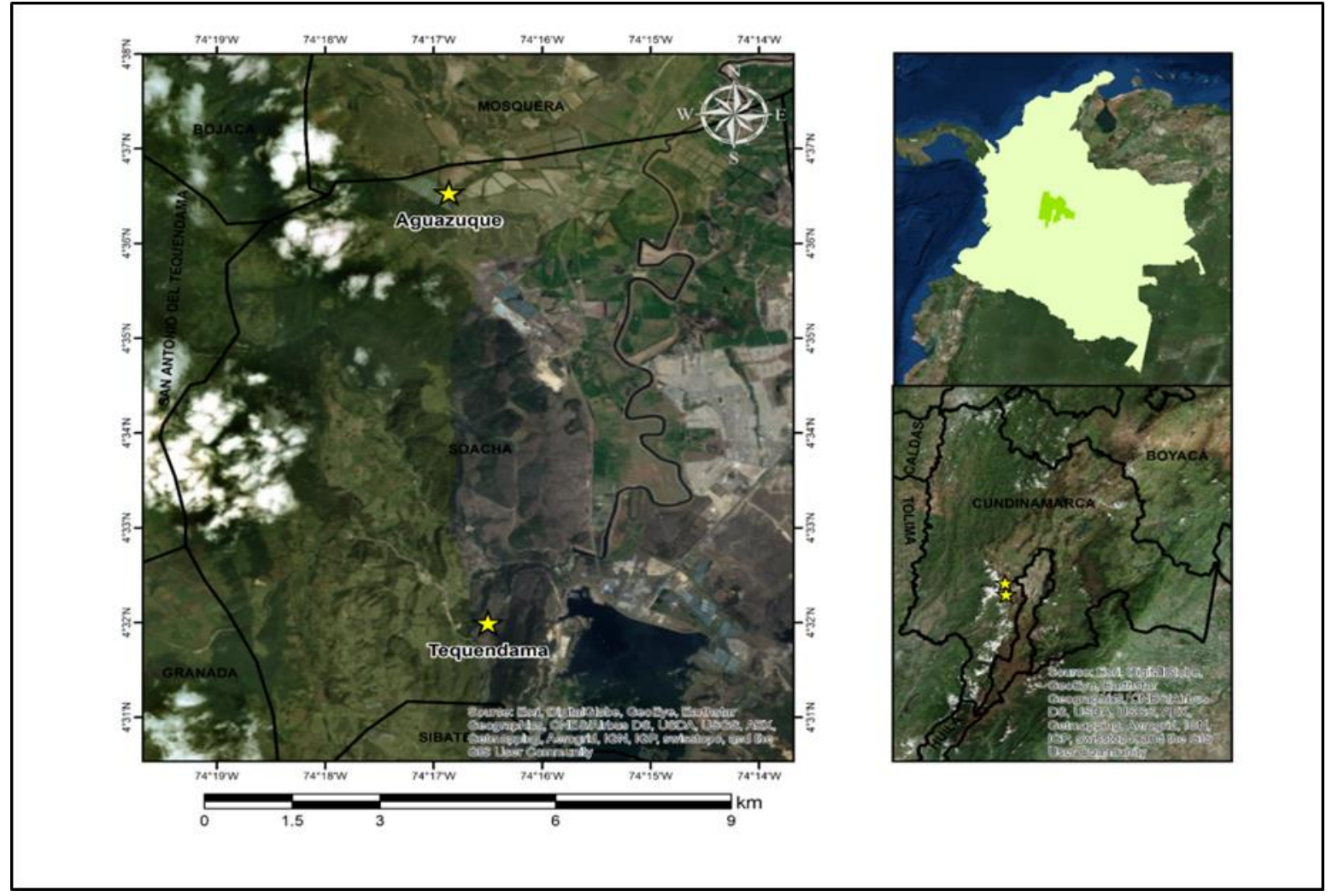

Fuente: Triana et al. (2019).

\section{Trabajo de campo}

En 2014 se excavaron columnas de ampliación de $50 \times 50 \mathrm{~cm}$ en el área donde finalizaron las excavaciones realizadas por Correal y Van der Hammen (1977) y Correal (1990). La metodología de excavación consistió en seleccionar cinco columnas en el sitio arqueológico de Tequendama, que se ubicaron teniendo en cuenta el datum determinado por los investigadores en la excavación y las unidades estratigráficas o áreas de ocupación de cada sitio, así como los reportes de la excavación con evidencias de áreas de fogón, concentraciones de artefactos, restos óseos humanos y de fauna y estructuras demarcadas por huellas de poste. Para el sitio arqueológico de Aguazuque, se tuvieron en cuenta las mismas características mencionadas, pero allí solo se seleccionaron cuatro columnas. Las excavaciones de estas se hicieron cada $5 \mathrm{~cm}$ o menos, según los rasgos o niveles estratigráficos que aparecían a lo largo de la excavación. Durante la excavación de los sitios se hallaron restos óseos humanos aislados, un entierro humano en el sitio de Tequendama y otro en Aguazuque, restos óseos de fauna, artefactos líticos y algunos fragmentos cerámicos.

\section{Selección de las muestras}

La selección de las muestras consistió en tomar una pequeña fracción de polvo de hueso (dos individuos) y extracción de esmalte de los terceros molares. Las muestras proceden de Tequendama y Aguazuque (tabla 1). En total se procesaron cuatro individuos, dos pertenecientes a la excavación de 2014, a los cuales se le realizó análisis en hueso y diente, y dos que forman parte de las excavaciones de años anteriores (Correal, 1990; Correal y Van

Triana-Vega, a.v.; Casar, I.; Morales, P. y Salinas, J. (2020). Análisis de Isótopos estables en restos óseos humanos y de fauna en los sitios arqueológicos del Holoceno temprano y medio Tequendama y Aguazuque (sabana de Bogotá, Colombia): Jangwa Pana, 19 (1), 
der Hammen, 1977), que se analizaron uno en hueso y otro en diente, respectivamente. Además de esto, se seleccionaron restos óseos de fauna que pertenecieron a especies como Odocoileus virginianus y Cavia sp. Con esto se tuvo la intención de identificar la dieta de estas especies, que son las más representativas en los grupos prehispánicos de la sabana de Bogotá. Se escogieron muestras de cada nivel estratigráfico en ambos sitios. Sin embargo, solo se pudieron procesar seis muestras en total por ambos sitios, cuatro pertenecientes a Odocoileus virginianus y Cavia sp. (tabla 2).

\section{Trabajo de laboratorio}

Las muestras para análisis de isótopos estables fueron seleccionadas, procesadas e interpretadas en el laboratorio de isótopos estables del Instituto de Geología de la Universidad Nacional Autónoma de México, con la dirección de los doctores Pedro Morales e Isabel Casar, y el acompañamiento técnico de Edith Cienfuegos y Francisco Otero.

El procesamiento de las muestras para el análisis isotópico en colágeno y bioapatita en hueso, tanto animal como humano, tuvo dos procedimientos. El primero consistió en realizar una limpieza de las muestras con agua destilada. Al secarse, se trituró con un martillo un trozo del hueso hasta que se obtuviera un fragmento que pesara aproximadamente $200 \mathrm{mg}$. Luego este fragmento fue triturado en un mortero de ágata para obtener un polvo en el que sus gránulos lograran atravesar un tamiz de 150-200 $\mu$.

El polvo obtenido fue vertido en un tubo de Eppendorf, del que luego se extrajo una pequeña cantidad para someterla a la prueba de FTIR, con el fin de reconocer si la muestra seleccionada era apta para al análisis isotópico. Con este análisis se evalúan los niveles de carbonatos que deben estar en un rango óptimo de 1 a $7 \%$, y el de cristalinidad, entre 3 y $4 \%$. Si la muestra se sale de estos rangos, no es recomendable realizar análisis isotópicos (Castaños et al., 2010).

El segundo procedimiento consistió en realizar una desmineralización a los huesos. Con este procedimiento se buscó disolver la bioapatita y evidenciar si la muestra poseía colágeno. Para esto se extrajo una muestra de hueso con un peso aproximado de $70 \mathrm{mg}$, que fue sometida a una solución de ácido clorhídrico $(\mathrm{HCl})$ al 0,5 molar. El ácido fue cambiado cada tres días durante tres semanas. Luego se revisó por medio de una aguja si la muestra poseía colágeno.

Para el análisis isotópico de $\delta^{13} \mathrm{C}$ y $\delta^{18} \mathrm{O}$ en esmalte dental, se extrajo el esmalte de los terceros molares de tres individuos humanos, usando una fresa con punta de diamante y de alta velocidad. De allí, se obtuvo un polvillo fino que posteriormente fue sometido al análisis de espectrometría de masas, junto con las otras muestras procesadas en el equipo de análisis de elementos Flash EA 1112, acoplado al espectrómetro de masas Thermo Finnigan Delta Plus XL, mediante una interfase ConFlo III y con una precisión de $0,2 \%$, en el laboratorio de espectrometría de masas de isótopos estables.

Lo anterior se realizó con el fin de analizar las proporciones de colágeno $\left(\delta^{13} \mathrm{C}\right.$ y $\left.\delta^{15} \mathrm{~N}\right)$ y bioapatita $\left(\delta^{13} \mathrm{C}\right.$ y $\left.\delta^{18} \mathrm{O}\right)$ en hueso. En cuanto al esmalte dental, se analizó la relación isotópica de $\delta^{13} \mathrm{C}$ y $\delta^{18} \mathrm{O}$, presentes en muestras de hueso duro, tanto animal como humano, y esmalte dental humano. Además, con el fin de estimar la relación isotópica entre el oxígeno fosfórico 
en la apatita de los restos óseos y el agua ambiental, se tomaron $60 \mathrm{ml}$ de agua de tres fuentes hídricas de la sabana de Bogotá, ubicadas en diferentes alturas.

\section{Declaración de aspectos éticos}

La excavación de los sitios arqueológicos y el material seleccionado para realizar los análisis de isótopos estables se realizaron bajo la autorización de la licencia arqueológica \#5764, aprobada por el Instituto Colombiano de Antropología e Historia (Icanh). Todos los procedimientos se realizaron conforme a lo establecido en la ley. Las muestras se procesaron en el laboratorio de isótopos estables en el Instituto de Geología de la Universidad Nacional Autónoma de México. Este laboratorio cumple con los estándares de medición y confiabilidad.

\section{Resultados}

En esta investigación se realizaron análisis de isótopos estables en cuatro individuos humanos y seis restos óseos de fauna. Estas muestras se ubican en periodos desde el Holoceno temprano 9000-7000 cal BP hasta el Holoceno medio 6600-4100 cal BP y finalmente Holoceno tardío 3800-2300 cal BP. Dichas muestras se seleccionaron teniendo en cuenta la cronología de los individuos. Es importante mencionar que solo se cuenta con dos fechas cronológicas absolutas de los individuos Agz rasgo 9 y Teq rasgo 6, ya que los otros dos individuos Agz 458-31 y Teq entierro 3 cuentan con una fecha asociada y no absoluta.

A partir de los resultados obtenidos, se puede observar que en Aguazuque y Tequendama los valores de $\delta^{13} \mathrm{C}$ son bajos, es decir, los recursos $\mathrm{C}_{3}$ son tanto animales como vegetales. Prevaleció un consumo de recursos vegetales $\mathrm{C}_{3}$ y animales, que, a su vez, consumieron pasturas bajo ese patrón fotosintético. En los valores de $\delta^{15} \mathrm{~N}$ se observa una dispersión amplia. Aunque los valores de proteína son bajos, se puede sugerir quizá un consumo asociado a proteína terrestre y vegetal (Triana, 2019).

Tabla 1. Muestras de restos óseos humanos seleccionadas para análisis de isótopos estables

\begin{tabular}{|c|c|c|c|c|c|c|c|c|c|}
\hline $\begin{array}{c}\text { Elemento } \\
\text { humano }\end{array}$ & Sitio & Subsistencia & Código & Sexo & Edad & $\begin{array}{c}\text { Fecha } \\
\text { AP sin } \\
\text { calibrar }\end{array}$ & $\begin{array}{c}\delta \\
{ }^{15} \text { Ncol }\end{array}$ & $\begin{array}{c}\delta \\
{ }^{13} \mathrm{Ccol}\end{array}$ & $\begin{array}{c}\delta \\
{ }^{13} \mathrm{Ca}\end{array}$ \\
\hline $\begin{array}{c}\text { Fragmento } \\
\text { óseo }\end{array}$ & Tequendama & $\begin{array}{c}\text { Caza- } \\
\text { recolección }\end{array}$ & $\begin{array}{c}\text { Teq } \\
\text { rasgo } 6\end{array}$ & $\mathrm{~F}$ & $\begin{array}{l}\text { Adulto } \\
\text { medio }\end{array}$ & $\begin{array}{c}6080 \pm \\
40\end{array}$ & 6,42 & $-19,99$ & $-12,94$ \\
\hline $\begin{array}{c}\text { Esmalte } \\
\text { m3 inf. } \\
\text { der. }\end{array}$ & Tequendama & $\begin{array}{c}\text { Caza- } \\
\text { recolección }\end{array}$ & $\begin{array}{c}\text { Teq } \\
\text { rasgo } 6 \\
\text { entierro } \\
1\end{array}$ & $\mathrm{~F}$ & $\begin{array}{l}\text { Adulto } \\
\text { medio }\end{array}$ & $\begin{array}{c}6080 \pm \\
40\end{array}$ & & & $-12,94$ \\
\hline $\begin{array}{c}\text { Fragmento } \\
\text { óseo }\end{array}$ & Tequendama & $\begin{array}{c}\text { Caza- } \\
\text { recolección }\end{array}$ & $\begin{array}{c}\text { Teq } \\
\text { entierro } \\
3 \\
\end{array}$ & $\mathrm{M}$ & $\begin{array}{l}\text { Adulto } \\
\text { joven }\end{array}$ & $\begin{array}{c}7500- \\
6000 \mathrm{AP}\end{array}$ & 7,14 & $-15,2$ & $-8,80$ \\
\hline $\begin{array}{c}\text { Fragmento } \\
\text { óseo }\end{array}$ & Aguazuque & $\begin{array}{l}\text { Caza- } \\
\text { recolección- } \\
\text { agricultura } \\
\text { incipiente }\end{array}$ & $\begin{array}{c}\text { Agz } \\
\text { rasgo } 9\end{array}$ & $\mathrm{~F}$ & $\begin{array}{l}\text { Adulto } \\
\text { joven }\end{array}$ & $\begin{array}{l}3600 \pm \\
40 \mathrm{AP}\end{array}$ & 6,85 & $-19,57$ & $-13,30$ \\
\hline
\end{tabular}




\begin{tabular}{|c|c|c|c|c|c|c|c|c|c|}
\hline $\begin{array}{c}\text { Esmalte } \\
\text { m3 sup. } \\
\text { der. }\end{array}$ & Aguazuque & $\begin{array}{c}\text { Caza- } \\
\text { recolección- } \\
\text { agricultura } \\
\text { incipiente }\end{array}$ & $\begin{array}{c}\text { Agz } \\
\text { rasgo 9 }\end{array}$ & F & $\begin{array}{c}\text { Adulto } \\
\text { joven }\end{array}$ & $\begin{array}{c}3601 \pm \\
40 \text { AP }\end{array}$ & & & $-12,35$ \\
\hline $\begin{array}{c}\text { Esmalte } \\
\text { m3 sup. } \\
\text { izq. }\end{array}$ & Aguazuque & $\begin{array}{c}\text { Caza- } \\
\text { recolección- } \\
\text { agricultura } \\
\text { incipiente }\end{array}$ & $458-31$ & Agz & M & $\begin{array}{c}\text { Adulto } \\
\text { joven }\end{array}$ & 2725 AP & & \\
\hline
\end{tabular}

Tabla 2. Muestras de fauna seleccionadas para análisis de isótopos estables

\begin{tabular}{|c|c|c|c|c|c|c|c|c|c|}
\hline Fauna & Especie & Sitio & $\begin{array}{l}\text { Fecha } \\
\text { AP sin } \\
\text { calibrar }\end{array}$ & \begin{tabular}{|c|}
$\delta 13$ \\
CaVPDB \\
(\%o) col \\
\end{tabular} & $\begin{array}{l}\delta 15 \\
\text { Nair } \\
(\%)\end{array}$ & $\begin{array}{c}\delta 13 \\
\text { CcVPDB } \\
\text { (\%o) apa }\end{array}$ & $\begin{array}{c}\delta^{18} \\
\text { OVPDB } \\
(\% 0)\end{array}$ & $\begin{array}{c}\delta^{18} \\
\text { OVsmow } \\
(\%)\end{array}$ & $\begin{array}{c}\delta^{18} \\
\text { OVsmov } \\
\text { (\%) agua } \\
\text { en } \\
\text { equilibrio }\end{array}$ \\
\hline $\begin{array}{c}\mathrm{Agz} \\
336-8 \\
\end{array}$ & \begin{tabular}{c}
\multicolumn{1}{c}{$\mathrm{Agz}$} \\
Odocoileus \\
virginianus
\end{tabular} & Aguazuque & 2745 & $-19,27$ & 3,43 & $-10,09$ & $-7,49$ & 23,18 & $-10,47$ \\
\hline $\begin{array}{c}\mathrm{Agz} \\
258- \\
11\end{array}$ & $\begin{array}{c}\mathrm{Agz} \\
\text { Odocoileus } \\
\text { virginianus }\end{array}$ & Aguazuque & 5045 & $-19,98$ & 4,03 & $-10,25$ & $-7,96$ & 23,43 & $-10,22$ \\
\hline $\begin{array}{c}\mathrm{Agz} \\
245-2\end{array}$ & $\begin{array}{c}\text { Agz } \\
\text { Odocoileus } \\
\text { virginianus }\end{array}$ & Aguazuque & 3865 & $-18,42$ & 4,30 & $-9,25$ & $-6,04$ & 24,68 & $-8,96$ \\
\hline $\begin{array}{c}\mathrm{Agz} \\
258-3\end{array}$ & $\begin{array}{l}\text { Agz Cavia } \\
\text { sp. }\end{array}$ & Aguazuque & 5045 & $-20,33$ & 2,60 & $-12,07$ & $-7,46$ & 23,22 & $-10,43$ \\
\hline $\begin{array}{c}\mathrm{Agz} \\
290-4 \\
\end{array}$ & Cavia sp. & Tequendama & 3410 & $-20,34$ & 2,69 & $-11,94$ & $-7,28$ & 23,40 & $-10,25$ \\
\hline $\begin{array}{c}\text { Teq } \\
162-3\end{array}$ & $\begin{array}{c}\text { Teq } \\
\text { Odocoileus } \\
\text { virginianus }\end{array}$ & Tequendama & 2240 & $-20,16$ & 4,15 & $-8,44$ & $-6,42$ & 24,29 & $-9,36$ \\
\hline
\end{tabular}

En la figura 1 se observa que las mujeres tienen un porcentaje un poco más bajo de proteína, aunque no es muy significativa. Quizás se deba por un acceso a recursos que represente bajos valores proteicos o que posiblemente su proteína sea obtenida de recursos vegetales. El individuo masculino de Tequendama (Teq rasgo 3) presenta una posible dieta mixta asociada a recursos $\mathrm{C}_{3} / \mathrm{C}_{4}$. Vale la pena aclarar que este valor es diferente a los otros valores obtenidos en los otros dos individuos femeninos, los cuales reflejan valores asociados a plantas $\mathrm{C}_{3}$. Por su lado, la fauna presenta una relación de la dieta determinada por plantas tipo $\mathrm{C}_{3}$; tanto el venado como el cavia presentan estos rangos de valores. 


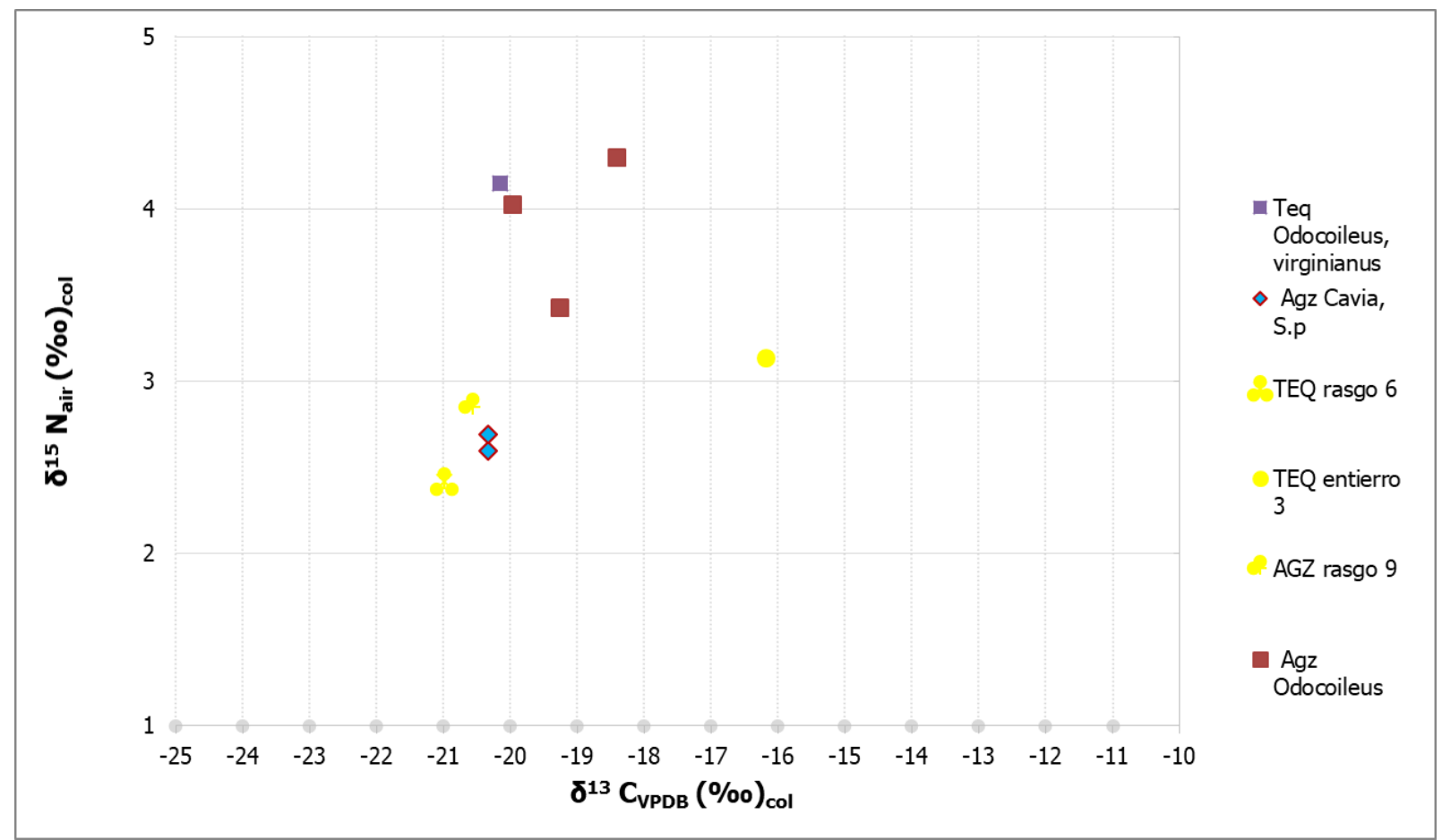

Figura 1. Relación $\delta^{15} \mathrm{~N}_{\text {air }}\left(\%\right.$ col $/ \delta^{13} \mathrm{C}_{\mathrm{VPDB}}(\% \mathrm{o}$ ) col humanos (amarillo) y fauna (otros colores) de Aguazuque y Tequendama

Con base en los resultados de los análisis de fauna se presenta una relación de la dieta determinada por plantas tipo $\mathrm{C}_{3}$; tanto el venado como el cavia presentan estos rangos de valores. También se puede pensar que la fauna que tiene valores altos de $\delta^{15} \mathrm{~N}_{\text {air }}$ (\%o) puede estar asociada a la diferencia entre especies y su tamaño. Igualmente puede deberse a que los valores isotópicos de cada sitio arqueológico varíen. Esto se puede pensar, ya que ambos animales son herbívoros y pueden representar estos valores diferenciales.

Así mismo, se evidencia que los valores entre especies son similares. Esto quiere decir que los ecosistemas son semejantes a pesar de que Aguazuque y Tequendama se encuentran en altitudes poco diferenciales. Además de esto, lo interesante es que tanto el venado (Odocoileus virginianus) y el cavia (cavia $\mathrm{Sp}$ ) ingieren agua con valores de oxígeno iguales y también presentan la misma dieta total (tabla 2).

En la línea de regresión de la figura 2 se observa que el individuo masculino Teq entierro 3 se acerca a una dieta mixta tipo $\mathrm{C}_{3} / \mathrm{C}_{4}$. Las dos mujeres pertenecen a cronologías diferentes: Tequendama (transición Holoceno temprano medio) y Aguazuque (transición Holoceno medio tardío). A pesar de esto, tienen una dieta completamente asociada a plantas tipo $\mathrm{C}_{3}$. 


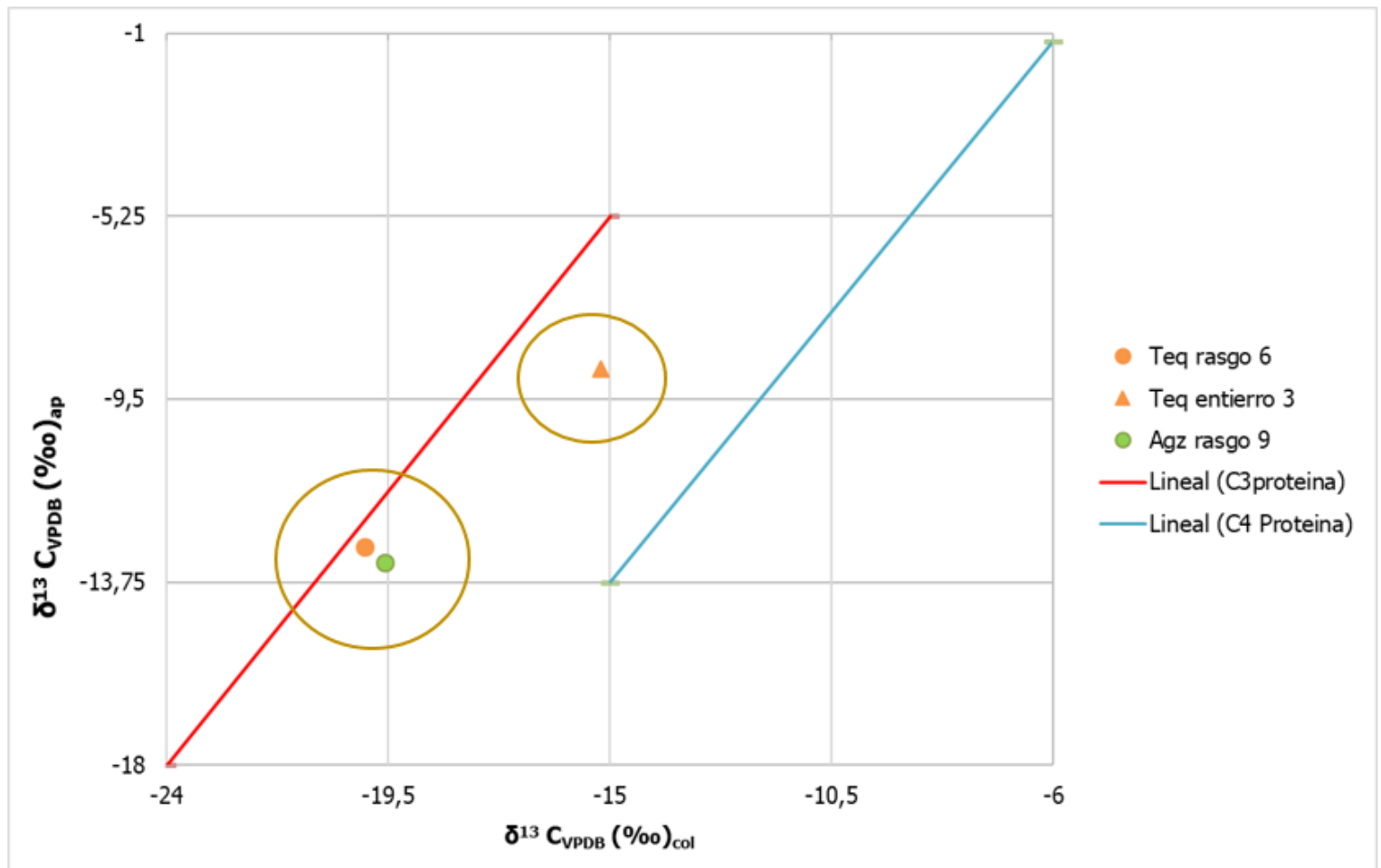

Figura 2. Relación $\delta^{13} \mathrm{C}_{\mathrm{VPDB}}(\% \mathrm{o})$ apa $/ \delta^{13} \mathrm{C}_{\mathrm{VPDB}}(\% \mathrm{o})$ col humanos Tequendama y Aguazuque, los círculos son mujeres, el triángulo es un hombre

Con base en lo anterior, y teniendo en cuenta la línea de regresión propuesta por Kellner y Schoeninger (2007), se puede evidenciar que el componente proteico de la dieta total está compuesto por animales que se encuentran consumiendo alimento tipo $\mathrm{C}_{3}$ (figura 2). Por otro lado, casi el $90 \%$ de la fuente de energía también proviene de recursos tipo $\mathrm{C}_{3}$, a diferencia del individuo Teq entierro 3 , que tiene una fuente de energía que es $\mathrm{C}_{3} / \mathrm{C}_{4}$, como se mencionó anteriormente.

En la figura 3 se observan valores variados en cuanto a la medición de oxígeno en hueso y esmalte. Por ejemplo, el individuo femenino de Aguazuque en su muestra de esmalte parece tener unos valores que se asocian a tierras más altas en edad de adolescencia. Por su lado, en los valores isotópicos en hueso se observa que este individuo en sus últimos años de vida posiblemente tuvo una movilidad hacia tierras un poco más bajas (Triana, 2019). No obstante, esta hipótesis debe ser complementada con otros datos teniendo en cuenta la edad del individuo. 


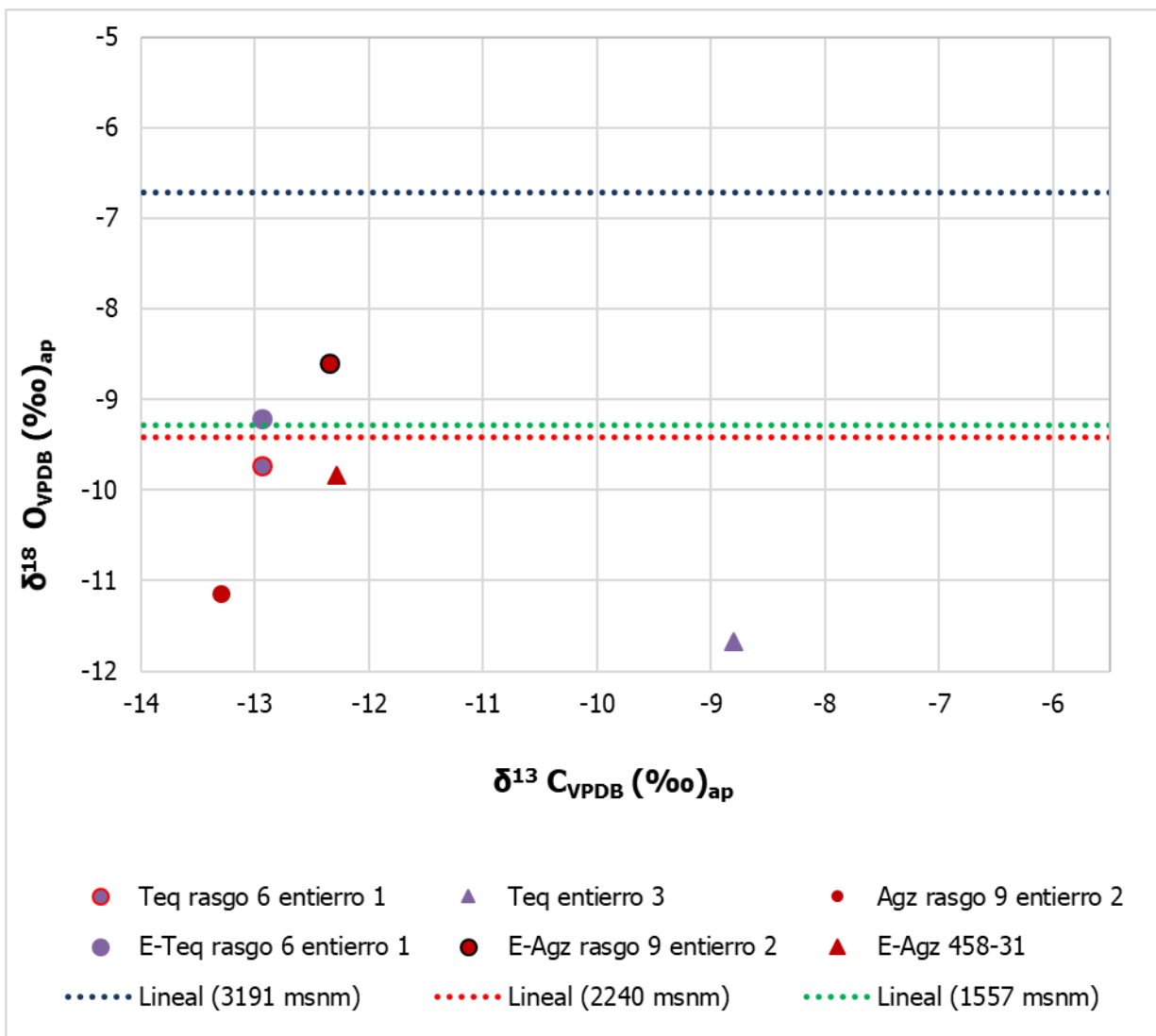

Figura 3. Relación $\delta^{18} \mathrm{OV}_{\text {smow }}\left(\%\right.$ ) apa $/ \delta^{13} \mathrm{C}_{\mathrm{VPDB}}(\%$ ) apa Humanos de Tequendama y Aguazuque, mediciones en esmalte y hueso

Por otro lado, el individuo entierro 3 masculino es el mismo que exhibe una dieta mixta. Con relación a los resultados de oxígeno se observa que se acerca a valores de tierras más bajas que todos los individuos. Por último, el individuo femenino de Tequendama sí presenta un acceso a los recursos hídricos similares a lo largo de su vida.

\section{Discusión}

En cuanto a la ecología isotópica, a partir de la información obtenida en las investigaciones paleoambientales, el tipo de vegetación disponible en diferentes periodos (Boom et al., 2001; Mora et al., 2002) y los datos existentes en isótopos estables en restos óseos humanos (Cárdenas, 2002; Delgado, 2018; Van der Hammen et al., 1990), se ha planteado que los valores elevados de $\delta^{13} \mathrm{C}$, entre los rangos de $-9 \mathrm{a}-15 \%$ son indicativos de la presencia de plantas tipo $\mathrm{C}_{4}$ y plantas CAM en los diferentes periodos del Holoceno (Delgado, 2018). Sin embargo, para los datos existentes en el Holoceno temprano se reporta la presencia de plantas tipo $\mathrm{C}_{3}$. Para el Holoceno medio, se ha registrado que las condiciones ambientales estaban asociadas a áreas abiertas. Se presentaron cambios en la temperatura y el clima. Las precipitaciones aumentaron, lo cual generó cambios en el ambiente ecológico para la sabana de Bogotá, y dio paso a una vegetación más arbórea y abierta, con una alta presencia de gramíneas (Marchant et al., 2002; Mora y Pratt, 2002). Lo anterior da cuenta de una posible combinación de dietas mixtas $\mathrm{C}_{3} / \mathrm{C}_{4}$ para los individuos de estos periodos, a juzgar por los valores isotópicos obtenidos (Cárdenas, 2002; Delgado, 2018). No obstante, los valores 
intermedios serían el producto del consumo indirecto de recursos $\mathrm{C}_{4}$. Las gramíneas podrían ser consumidas por los animales cazados por estos individuos.

Para periodos más tardíos, los valores obtenidos de $-12,8$ hasta $-9,7 \%$ o aproximadamente corresponden con una dieta basada en maíz, teniendo en cuenta la información existente en los datos obtenidos de los isótopos estables en los individuos de estos periodos (Cárdenas, 2002; Delgado, 2018) y en las condiciones paleoambientales, la disponibilidad de plantas reportadas y el registro arqueológico asociado a estos periodos de ocupación (Van der Hammen, 1992).

Con base en los resultados obtenidos en la presente investigación, y con la caracterización isotópica que se ha reportado para la sabana de Bogotá, se evidencia que los dos individuos femeninos obtenidos en la excavación del 2014 presentan valores proteicos bajos, posiblemente los valores de $\delta^{15} \mathrm{~N}$ más bajos en comparación con los datos de Cárdenas (2000) en individuos femeninos de ambos sitios. Se podría pensar que los valores isotópicos de estos dos individuos fueron obtenidos por el consumo de plantas. En general, su dieta está enriquecida con fuentes de energía $\mathrm{C}_{3}$, lo cual es consecuente con la caracterización isotópica que existe en los diferentes momentos entre el Holoceno temprano, medio y tardío (Triana, 2019).

En cuanto al individuo masculino de Tequendama, se observan valores de $\delta^{15} \mathrm{~N}$ diferentes en comparación con los datos obtenidos por Cárdenas (2000) en individuos masculinos. En la fracción isotópica de este individuo, se puede sugerir que tuvo un acceso diferente tanto a proteína animal como a la obtenida de plantas $\mathrm{C}_{3}$. Además, los valores de oxígeno de este individuo son diferentes en comparación con los valores de los dos individuos femeninos.

Los datos obtenidos en el individuo entierro 3 son interesantes debido a que se sale de los valores que se esperarían encontrar en grupos que pertenecen al Holoceno medio, donde se ha reportado una ecología isotópica asociada a plantas tipo $\mathrm{C}_{3}$; es decir, no se espera encontrar valores de una dieta mixta $\mathrm{C}_{3} / \mathrm{C}_{4}$ para este periodo. Este resultado arroja varias posibles hipótesis con respecto a la movilidad, el uso, el acceso y la disponibilidad de los recursos en la sabana de Bogotá (Triana, 2019).

Respecto a los datos de fauna, se evidencia una dieta similar en ambas especies muestreadas en diferentes periodos de ocupación de los sitios. Con base en la ecología isotópica de la sabana, los valores obtenidos concuerdan con la disponibilidad ambiental para los periodos analizados (Triana, 2019). Los datos isotópicos de la fauna proporcionan una importante información a la ecología isotópica de la sabana de Bogotá. Sin embargo, es necesario desarrollar más estudios en este aspecto que permitan ampliar dicha información.

\section{Conclusiones}

Esta investigación ofrece datos de un muestreo limitado en los grupos de cazadoresrecolectores de la sabana de Bogotá, especialmente de los sitios de Tequendama y Aguazuque. No obstante, la información obtenida abre una ventana para indagar el acceso a los recursos de estas poblaciones del pasado. Los datos obtenidos de valores proteicos bajos en mujeres, una dieta mixta en un individuo masculino y una dieta similar y con acceso a las 
mismas fuentes hídricas de la fauna nos dan cuenta de una nueva información que permite profundizar a escala local la disponibilidad de los recursos. Es necesario ampliar estos datos con individuos de diferentes periodos de ocupación y sexo. Así mismo, obtener información de otras especies y fuentes hídricas en otras altitudes permitirá tener un panorama amplio acerca de las formas de vida de los grupos de cazadores-recolectores.

\section{Agradecimientos}

La investigación se desarrolló gracias al apoyo y la financiación de la Fundación Nacional de Investigaciones Arqueológicas (FIAN), al Instituto Colombiano de Antropología e Historia (Icanh) y a la fundación CEIBA por la beca doctoral; a la Universidad de los Andes, en especial a Carl Langebaek, y al Instituto de Ciencias Naturales de la Universidad Nacional, en especial a Germán Peña, por el constante acompañamiento y el apoyo en el uso de los laboratorios y equipos; a la Universidad Nacional Autónoma de México (UNAM), al Instituto de Geología y particularmente al laboratorio de Isótopos estables, por todos los análisis e interpretaciones de los datos, especialmente a los técnicos Edith Cienfuegos y Francisco Otero, por el constante acompañamiento; a los dueños y a todo el personal en general de las fincas Tequendama y Aguazuque, por su constante apoyo y confianza durante la excavación de los sitios.

\section{Referencias}

Boom, A., Mora, G., Cleef, A. y Hooghiemstra, H. (2001). High altitude C4 grasslands in the northern Andes: relicts from glacial conditions? Review of Palaeobotany and Palynology, 115(3-4), 147-160. DOI: https://doi.org/10.1016/S0034-6667(01)00056-2

Cárdenas, F. (2002). Datos sobre la alimentación prehispánica en la sabana de Bogotá, Colombia. Bogotá: Instituto Colombiano de Antropología e Historia.

Castaños, J., Murelaga, X., Castellanos, I., Olazábal, A. y Zuluaga, M. (2010). Evaluación del grado de diagénesis en huesos fósiles mediante espectroscopía de infrarrojos. Geogaceta, 49, 11-14.

Correal, G. y Van der Hammen, T. (1977). Investigaciones arqueológicas en los abrigos rocosos del Tequendama: 12.000 años de historia del hombre y su medio ambiente en la altiplanicie de Bogotá. Bogotá: Fondo de Promoción de la Cultura del Banco Popular.

Correal, G. (1990). Aguazuque: evidencias de cazadores, recolectores y plantadores en la altiplanicie de la Cordillera Oriental. Bogotá: Fundación de Investigaciones Arqueológicas Nacionales, Banco de la República.

Delgado, M. (2012). Dental and craniofacial diversity in the northern Andes, and the early peopling of South America. En L. Miotti, M. Saleme, N. Flegenheimer y T. Goebel (Eds.), Southbound: Late Pleistocene peopling of Latin America. Texas: Center for the Study of the First Americans (pp. 33-38). Texas: Department of Anthropology, University of Texas.

Delgado, M. (2017). Holocene population history of the Sabana de Bogotá region, Northern South America: An assessment of the craniofacial shape variation. American Journal of Physical Anthropology, 162, 350-369. DOI: https://doi.org/10.1002/ajpa.23124

Delgado, M. (2018). Stable isotope evidence for dietary and cultural change over the Holocene at the Sabana de Bogotá region, Northern South America. Archaeological

Triana-Vega, a.v.; Casar, I.; Morales, P. y Salinas, J. (2020). Análisis de Isótopos estables en restos óseos humanos y de fauna en los sitios arqueológicos del Holoceno temprano y medio Tequendama y Aguazuque (sabana de Bogotá, Colombia): Jangwa Pana, 19 (1), 10 - 22. Doi: https://doi.org/10.21676/16574923.3432 
Anthropological Sciences, 10, 817-832. DOI: https://doi.org/10.1007/s12520-0160403-3

Kellner, C. y Schoeninger, M. (2007). A simple carbon isotope model for reconstructing prehistoric human diet. American Journal of Physical Anthropology, 133, 1112-1127. DOI: https://doi.org/10.1002/ajpa.20618

Marchant, R., Behling, H., Berrío, J., Cleef, A., Duivenvoorden, J., Hooghiemstra, H. et al. (2002). Pollen-based biome reconstructions for Colombia at 3000, 6000, 12000, 15000 and 18000 14C yr ago: Late Quaternary tropical vegetation dynamics. Journal of Quaternary Science 17, 113-129.

Mora, G. y Pratt, L. (2002). Carbon isotopic evidence from paleosols for mixed $\mathrm{C}_{3} / \mathrm{C}_{4}$ vegetation in the Bogotá Basin, Colombia. Quaternary Science Reviews, 21(8), 985995. DOI: https://doi.org/10.1016/S0277-3791(01)00079-8

Triana, A. V. y Salinas, J. (2014). El uso de las plantas y las prácticas alimentarias a partir del análisis de macro y microrrestos en el precerámico de la cordillera oriental de Colombia (sitios arqueológicos de Tequendama, Aguazuque y Zipacón). Autorización de intervención arqueológica \#4152. Bogotá.

Triana, A. V. (2019). Dieta y acceso a recursos determinadas a partir del sexo en grupos de cazadores recolectores de la sabana de Bogotá durante el Holoceno temprano y medio (tesis inédita de doctorado). Universidad de los Andes, Bogotá, Colombia.

Triana, A. V., Sedovb, S., Salinas-Acero, J., Carvajal-Contreras, D., Moreano, C., TovarReyes, M. et al. (2019). Environmental reconstruction spanning the transition from hunter/gatherers to early farmers in Colombia: paleopedological and archaeological indicators from the pre-ceramic sites Tequendama and Aguazuque. Quaternary International, 516,175-189. DOI: https://doi.org/10.1016/j.quaint.2018.09.048

Van der Hammen, T., Urrego, G. y Klinken, G. (1990). Isótopos estables y dieta del hombre prehistórico en la sabana de Bogotá (un estudio inicial). Boletín Arqueología FIAN, 5(2), 3-10.

Van der Hammen, T. (1992). Historia, ecología y vegetación. Bogotá: Corporación Colombiana para la Amazonia, Araracuara. 